\title{
2
}

\section{CATALYTIC REACTIONS IN THE}

\section{SOLAR NEBULA}

\author{
E. ANDERS, R. HAYATSU, AND M. H. STUDIER
}

Organic compounds in meteorites seem to have formed by Fischer-Tropschtype, catalytic reactions of $\mathrm{CO}, \mathrm{H}_{2}$, and $\mathrm{NH}_{3}$ in the solar nebula, at $360-400 \mathrm{~K}$ (and 4-10) $\times 10^{-6} \mathrm{~atm}$. The onset of these reactions was triggered by the formation of catalytically active grains of magnetite and serpentine at these temperatures.

Laboratory experiments show that the Fischer-Tropsch reaction gives a large kinetic isotope fractionation of $c^{12} / c^{13}$, duplicating the hitherto unexplained fractionation in meteorites. All of the principal compound classes in meteorites are produced by this reaction, or a variant involving a brief excursion to higher temperatures. (1) normal, mono-, and dimethylalkanes; (2) arenes and alkylarenes; (3) dimeric isoprenoids from $C_{9}$ to $C_{14}$; (4) purines and pyrimidines, such as adenine, guanine, uracil, thymine, xanthine, etc.; (5) amino acids, including tyrosine and histidine; (6) porphyrin-like pigments; (7) aromatic polymer with $-\mathrm{OH}$ and - $\mathrm{COOH}$ groups.

These reactions may also have played a major role in the evolution of life: first, by converting carbon to a sufficiently non-volatile form to permit its accretion by the inner planets; second, by synthesizing organic compounds on the primitive planets whenever $\mathrm{CO}, \mathrm{H}_{2}, \mathrm{NH}_{3}$, and clay or magnetite particles came together at the right temperature. Similar reactions in other solar nebulae may be the source of interstellar molecules, as first suggested by G. H. Herbig. Ten of the twelve polyatomic interstellar molecules have in fact been see in these synthesis or in meteorites.

To his regret, Dr. Anders has not been able to find the time to write his contribution. The present abstract summarizes the paper published in "Origins of Life" (1974), 5, 57, a revised version of the authors" article in Science, (1973), 182, 781. This fundamenta1 contribution could not be totally ignored here. 